\title{
Editorial
}

\section{Dynamical Aspects of Initial/Boundary Value Problems for Ordinary Differential Equations}

\author{
Jifeng Chu, ${ }^{1}$ Juntao Sun, ${ }^{2}$ Patricia J. Y. Wong, ${ }^{3}$ and Yonghui Xia ${ }^{4}$ \\ ${ }^{1}$ Department of Mathematics, College of Science, Hohai University, Nanjing 210098, China \\ ${ }^{2}$ School of Science, Shandong University of Technology, Zibo, Shandong 255049, China \\ ${ }^{3}$ School of Electrical and Electronic Engineering, Nanyang Technological University, 50 Nanyang Avenue, Singapore 639798 \\ ${ }^{4}$ Department of Mathematics, Zhejiang Normal University, Jinhua 321004, China
}

Correspondence should be addressed to Jifeng Chu; jifengchu@126.com

Received 19 February 2013; Accepted 19 February 2013

Copyright (C) 2013 Jifeng Chu et al. This is an open access article distributed under the Creative Commons Attribution License, which permits unrestricted use, distribution, and reproduction in any medium, provided the original work is properly cited.

Dynamical aspects of initial/boundary value problems for ordinary differential equations have become a rapidly growing area of research in the theory of differential equations and dynamical systems and have gathered substantial research interests during the last decades. The attractiveness of this field not only is derived from theoretical interests but also is motivated by the insights that such dynamical aspects could reveal in several phenomena observed in applied sciences.

The current special issue places its emphasis on the study of the dynamical aspects of initial/boundary value problems for ordinary differential equations. Call for papers has been carefully prepared by the guest editors and posted on the journals web page, which has attracted many researchers to submit their contribution on wide topics such as oscillation theory, delay differential equation, impulsive differential equation, multipoint boundary value problems, stochastic mutualism system, chaotic system, homoclinic solutions, Hamiltonian systems, stability and bifurcation, exponential extinction, singular elliptic problem, nonuniform exponential contraction and dichotomy.

All manuscripts submitted to this special issue went through a thorough peer-refereeing process. Based on the reviewers' reports, we collect twenty-five original research articles by more than fifty active international researchers in differential equations and from different countries such as Korea, China, Malaysia, Singapore, Czech Republic, Turkey, Slovenia, India, and USA. Besides, one survey on recent results for the existence of singular periodic problems is also contained.

It is certainly impossible to provide in this short editorial note a more comprehensive description for all articles in this special issue. However, the team of the guest editors believes that the results included reflect some recent trends in research and outline new ideas for future studies of dynamical aspects of initial/boundary value problems for ordinary differential equations.

\section{Acknowledgment}

We would like to express our gratitude to the authors who have submitted papers for consideration. Thanks also are given to the many reviewers whose reports are important for us to make the decisions. All the participants have made it possible to have a very stimulating interchange of ideas. We would also like to thank the editorial board members of this journal, for their support and help throughout the preparation of this special issue.

Jifeng Chu Juntao Sun Patricia J. Y. Wong Yonghui Xia 


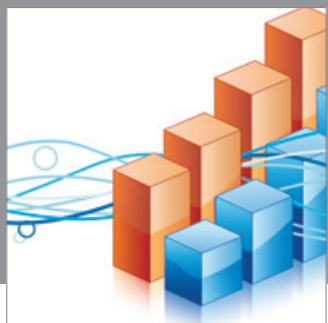

Advances in

Operations Research

mansans

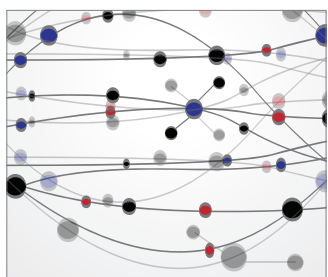

The Scientific World Journal
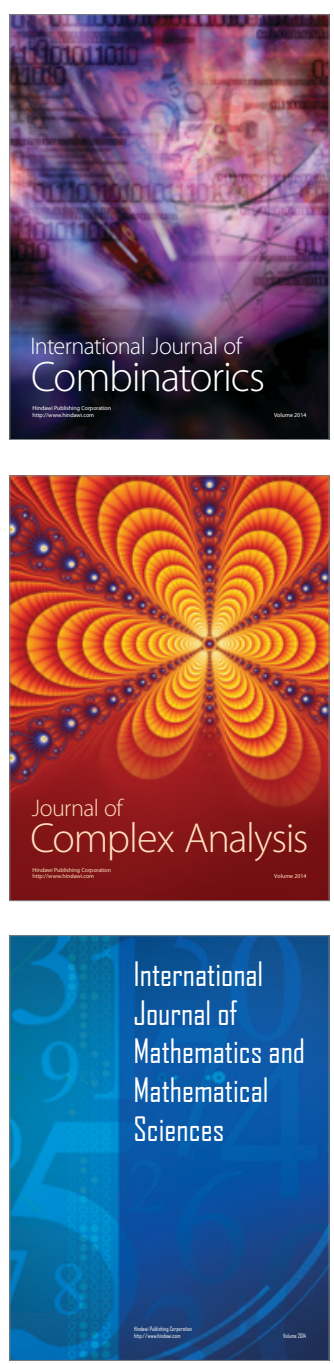
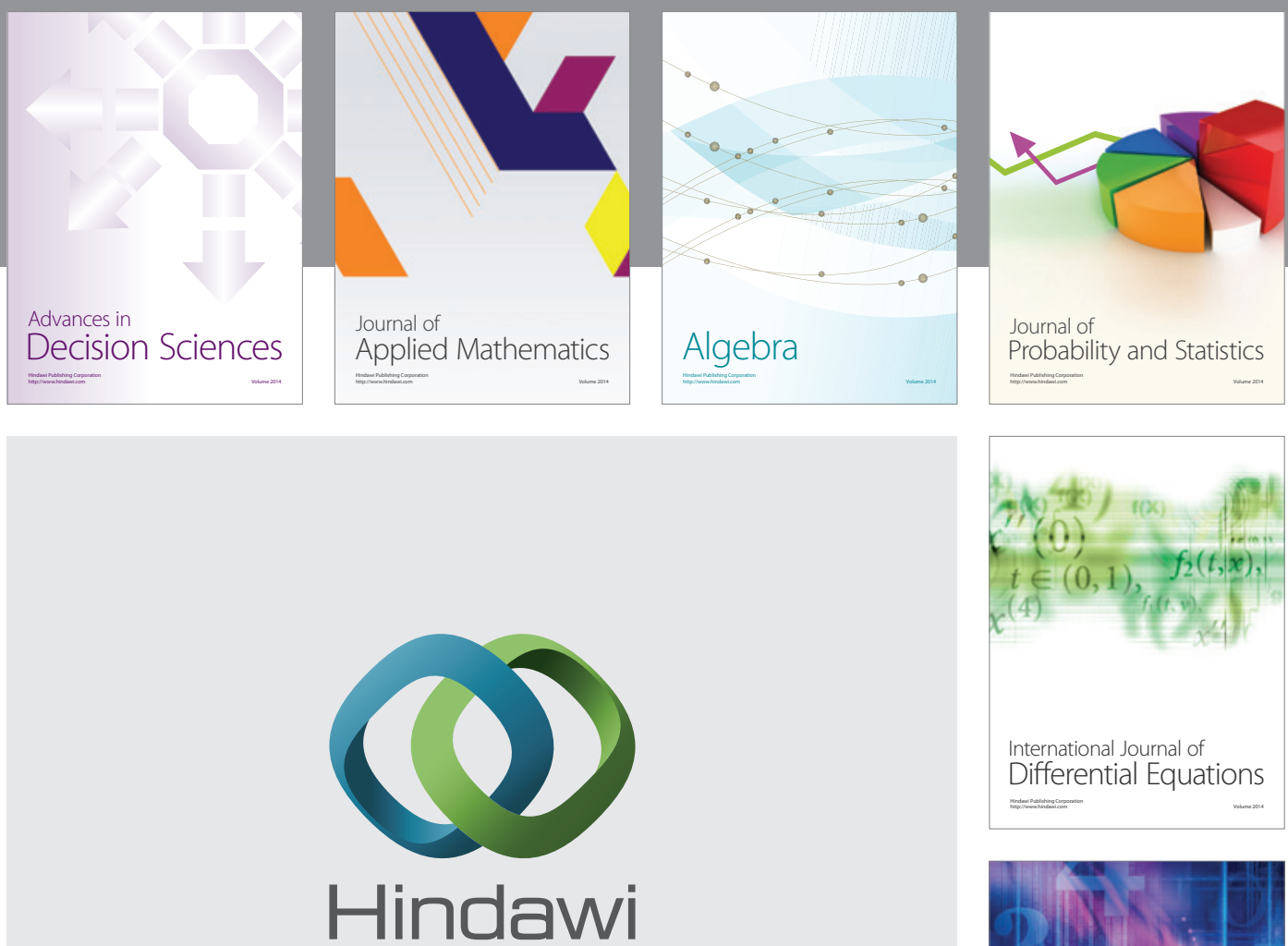

Submit your manuscripts at http://www.hindawi.com
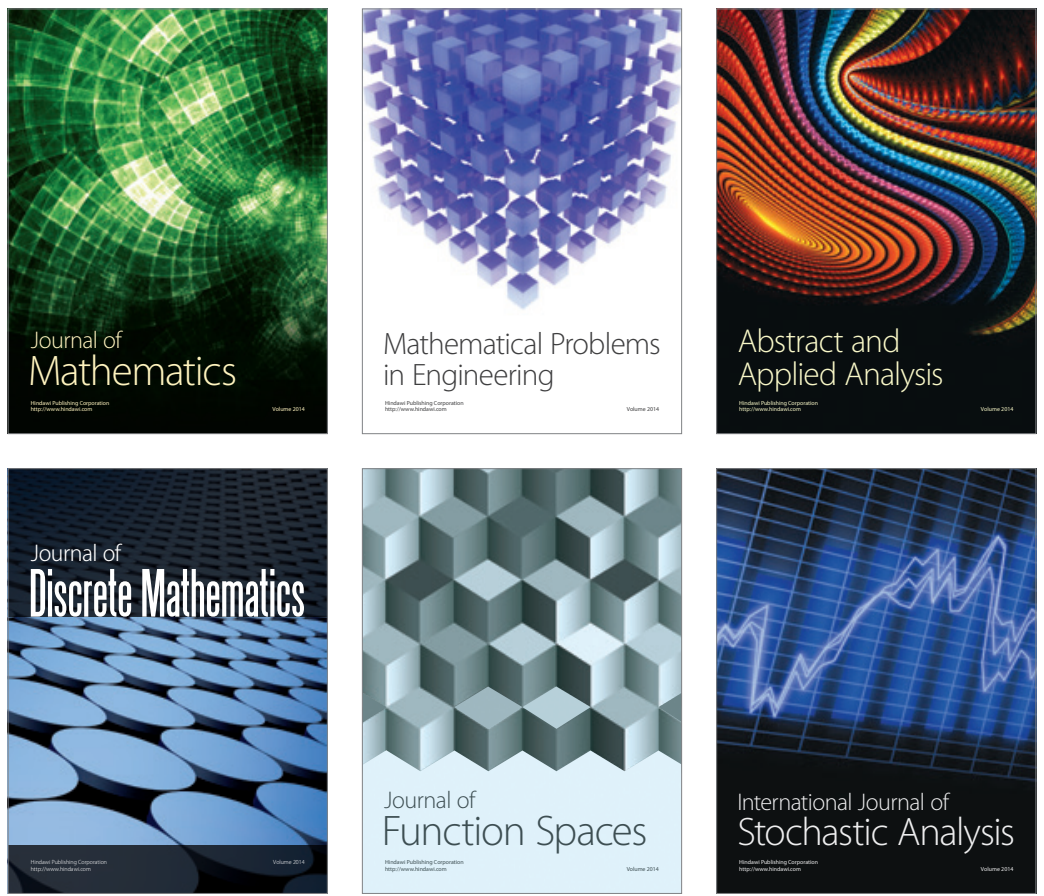

Journal of

Function Spaces

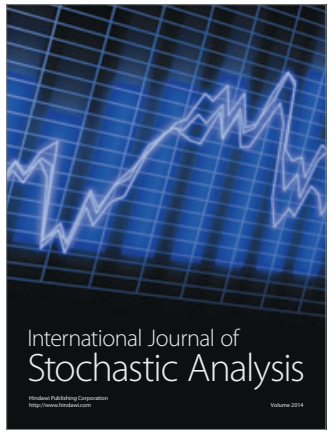

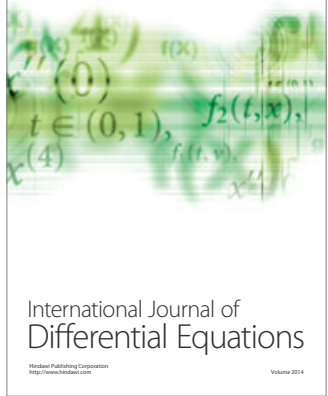
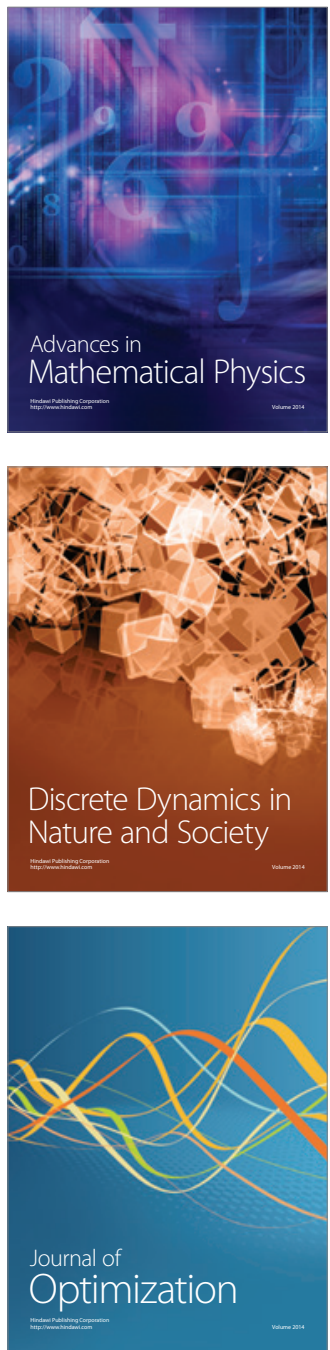